\title{
Picosecond time scale imaging of mechanical contacts
}

\author{
Thomas Dehoux $^{\mathrm{a}, *}$, Oliver B. Wright ${ }^{\mathrm{a}}$, Roberto Li Voti ${ }^{\mathrm{b}}$ \\ ${ }^{a}$ Division of Applied Physics, Graduate School of Engineering, Hokkaido University, Sapporo 060-8628, Japan \\ ${ }^{\mathrm{b}}$ Dipartimento di Energetica, Sapienza Università di Roma, Via A. Scarpa 16, Roma 00161, Italy
}

\section{A R T I C L E I N F O}

\section{Article history:}

Received 18 June 2009

Received in revised form 7 August 2009

Accepted 7 August 2009

Available online 14 August 2009

\section{Keywords:}

Contact mechanics

Nanoindentation

Picosecond ultrasonics

Thermoreflectance

\begin{abstract}
A B S T R A C T
By means of an ultrafast opto-acoustic technique we study the nanoindentation of thin chromium films on sapphire substrates using a ceramic ball bearing. Acoustic pulses at $\sim 40 \mathrm{GHz}$ returning from the filmindenter interface allow the film indentation profiles to be probed to sub-nanometer resolution over contact areas $\sim 25 \mu \mathrm{m}$ in radius. The deformation of the films during loading is thereby revealed. Furthermore, thermal wave imaging of the contact at megahertz frequencies is simultaneously achieved.
\end{abstract}

(C) 2009 Elsevier B.V. All rights reserved.

\section{Introduction}

The trend for device miniaturization requires the development of characterization techniques for the mechanical properties of nanostructures, especially thin films. Nanoindentation has provided a means to measure both elastic and plastic properties [1]. The use of sharp probes, such as pyramidal indenters or the tips in atomic force microscopes (AFM), offers nanometer lateral resolution determined by the indenter radius [2]. Owing to the localized force, plastic deformation occurs immediately as the sample is indented. In contrast to the case of spherical indenters [3], these sharp-tip techniques cannot be used to separately study elastic and plastic behaviour. Plastic deformation leads to pile-up or sink-in near the edges of the contact, inducing errors in the measurement of hardness or elasticity [1]. To quantify such effects, AFM or electron microscope imaging of the indented surface can be performed after the indenter is withdrawn. However, this does not permit detailed measurement of plastic flow during loading.

Attention has therefore been focused on imaging during loading. In particular, ultrasonic imaging up to megahertz frequencies in contact mechanics allows access to the interfacial stiffness or the contact pressure [4-8]. However, with a resolution of the order of the acoustic wavelength $(\sim 0.1 \mathrm{~mm})$ at the typical frequencies used $(\sim 30 \mathrm{MHz})$, one cannot effectively probe contacts of nanoscale thickness. In this paper we describe a profiling technique for nanoscale mechanical contacts that implements the generation and detection of ultrasound with ultrashort optical pulses [9-11],

\footnotetext{
* Corresponding author.

E-mail address: dehouxthomas@yahoo.fr (T. Dehoux).
}

offering acoustic frequencies up to and above $1 \mathrm{THz}$, sub-micron acoustic wavelengths, and $\sim 1 \mu \mathrm{m}$ lateral resolution by optical focusing to a diffraction limited spot. Furthermore, we show how this technique provides access to thermal properties through imaging $\mathrm{MHz}$ variations in thermoreflectance.

\section{Contact characterization}

We prepared two samples consisting of polycrystalline $\mathrm{Cr}$ films of thicknesses $110 \mathrm{~nm}$ and $215 \mathrm{~nm}$ deposited by RF (radio frequency) sputtering on ( 00001$)$ sapphire substrates of thickness $0.8 \mathrm{~mm}$. The samples lie horizontally on a rigid holder pierced with a $3.5 \mathrm{~mm}$ diameter hole for optical access. A sintered silicon nitride ball bearing of radius $R=2.98 \mathrm{~mm}$ is pressed against the $\mathrm{Cr}$ film using a high precision displacement stage. The load, monitored with a load cell, is stable to $5 \%$ during the experiments. Relevant physical properties are the longitudinal sound velocity $V_{n}$, Poisson's ratio $v_{n}$ and Young's modulus $E_{n}$, with $n=i, f, s$ referring to the indenter, film and substrate, respectively. We use the following values: $V_{i}=10.6 \mathrm{~km} \mathrm{~s}^{-1}, V_{f}=6.65 \mathrm{~km} \mathrm{~s}^{-1}, V_{s}=11.1 \mathrm{~km} \mathrm{~s}^{-1}, v_{i}=0.29$, $v_{f}=0.21, v_{s}=0.23, E_{i}=320 \mathrm{GPa}, E_{f}=280 \mathrm{GPa}$ and $E_{s}=440 \mathrm{GPa}[10-$ 12].

In order to study phonon transmission through a nanoscale heterogeneous interface, we used highly polished surfaces. The surface roughnesses of the films and the sphere were determined using an AFM with a $40 \mathrm{~nm}$ step. Typical line traces are shown in Fig. 1. From the AFM images, we determine the distributions of asperity heights for each sample, as plotted in Fig. 2. We obtain a good fit of these distributions to Gaussian functions. The rms roughnesses thus obtained, defined as one standard variation from 
(a)

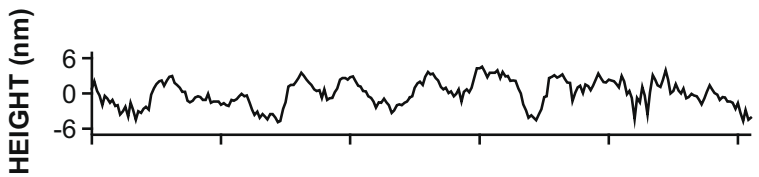

(b)

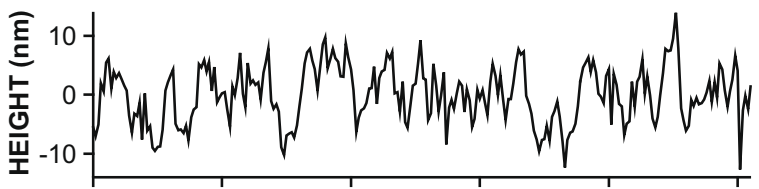

(c)

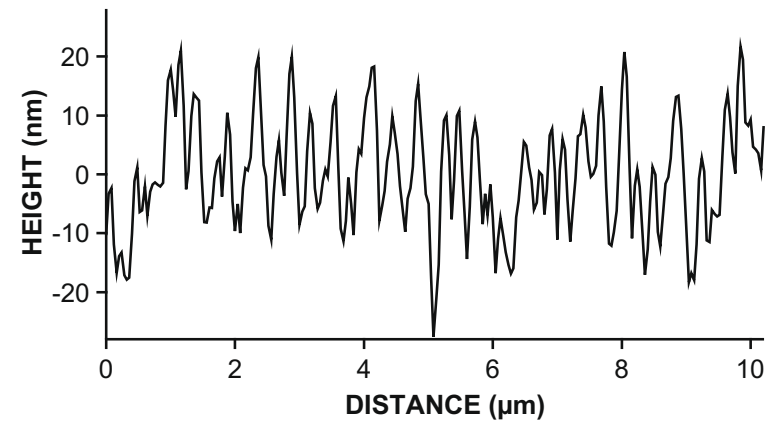

Fig. 1. Typical AFM traces measured in: (a) the $110 \mathrm{~nm} \mathrm{Cr}$ film, (b) the $215 \mathrm{~nm} \mathrm{Cr}$ film and (c) the $\mathrm{Si}_{3} \mathrm{~N}_{4}$ spherical indenter.
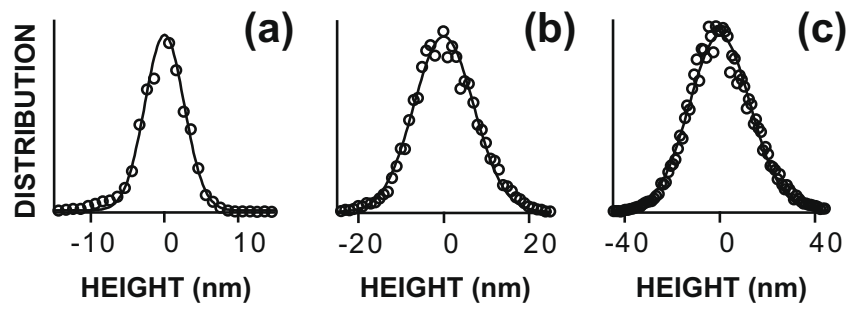

Fig. 2. Measured (circles) asperity height probability distributions and Gaussian function fit (lines) for: (a) the $110 \mathrm{~nm} \mathrm{Cr}$ film, (b) the $215 \mathrm{~nm} \mathrm{Cr}$ film and (c) the $\mathrm{Si}_{3} \mathrm{~N}_{4}$ spherical indenter.

the mean value, are $\sigma_{f 1}=3 \mathrm{~nm}, \sigma_{f 2}=7 \mathrm{~nm}$ and $\sigma_{i}=13 \mathrm{~nm}$ for the $110 \mathrm{~nm}$ film, the $215 \mathrm{~nm}$ film and the indenter, respectively. The rms roughness of the bare sapphire substrate (not shown) is $\sim 1 \mathrm{~nm}$. Since we measured distributions that are Gaussian, we can neglect asymmetric effects such as skewness and tortuosity [13]. Therefore the asperity geometry and the behaviour of the contact can be predicted by standard theories [14-16].

The generic Hertz theory describes the contact between a rigid sphere of radius $R$ and a flat elastic half-space, pressed onto each other with a force $F$. The pressure at the contacting interface $p(r)$ is distributed over a circular contact area of radius $a$ as follows [14]:

$p(r)=p_{0}\left[1-\left(\frac{r}{a}\right)^{2}\right]^{1 / 2}$,

where $r$ is the radial coordinate and $p_{0}$ is the maximum pressure at the center of the contact $(r=0)$ :

$p_{0}=\frac{3 F}{2 \pi a^{2}}$.

At such a pressure $p_{0}$, the rigid sphere penetrates into the sample down to a depth $\delta_{0}$ at $r=0$ :

$\delta(r=0)=\delta_{0}=\frac{a^{2}}{R}$.
To describe accurately our indentation conditions, we need to include the elasticities of the $\mathrm{Cr}$ film and $\mathrm{Si}_{3} \mathrm{~N}_{4}$ sphere. Since the $\mathrm{Cr}$ film thickness is very thin compared to $a$ (typically $\sim 30 \mu \mathrm{m}$ in our experiment), we assume the pressure distribution at the surface of the substrate is not modified by the metal layer. So we consider the simplified system of a $\mathrm{Al}_{2} \mathrm{O}_{3}$ half-space indented by a $\mathrm{Si}_{3} \mathrm{~N}_{4}$ sphere [14]:

$a=\left(\frac{3 F R}{4 E^{*}}\right)^{1 / 3}$

where

$E^{*}=\left[\frac{1-v_{i}^{2}}{E_{i}}+\frac{1-v_{s}^{2}}{E_{s}}\right]^{-1}$

is the effective Young modulus accounting for the elasticities of both the indenter and the substrate. Empirical models $[17,18]$ were proposed to modify $E^{*}$ to include the influence of the $\mathrm{Cr}$ film. In our case of a soft layer on a hard substrate,

$\frac{\left(1-v_{s}\right) E_{f}}{\left(1-v_{f}\right) E_{s}}=0.6<1$,

the value of $a$ is expected to be slightly (by less than 5\%) larger compared to the no-layer case [19]. As seen from Eqs. (2) and (3), the contact pressure should then decrease, and the total indentation depth should increase accordingly. This relatively small effect is not considered here. The quantity $\delta_{0}$ of Eq. (3) is in this case not the indentation of the $\mathrm{Cr}$ film, but the approach of distant points in the normal direction of the whole $\mathrm{Al}_{2} \mathrm{O}_{3} / \mathrm{Si}_{3} \mathrm{~N}_{4}$ system.

The surface roughness may also modify the pressure distribution and prevent the application of Hertz theory. Provided the scaled roughness $\alpha=\sigma / \delta_{0} \ll 1$, where $\sigma=\left(\sigma_{f}^{2}+\sigma_{i}^{2}\right)^{1 / 2}$ is the combined roughness, Hertz theory remains valid [14]. In the present case, $\sigma=15 \mathrm{~nm}$ for the rougher film and $\delta_{0} \approx 180 \mathrm{~nm}$ at the lowest load $F=1.1 \mathrm{~N}$. So $\alpha<0.08 \ll 1$, and Hertz theory applies increasingly well as the load increases.

\section{Picosecond ultrasonics}

Picosecond longitudinal acoustic pulses are generated in the $\mathrm{Cr}$ films at the $\mathrm{Al}_{2} \mathrm{O}_{3}-\mathrm{Cr}$ interface with linearly polarized pump pulses of duration $200 \mathrm{fs}$ from a Ti:Sapphire mode-locked laser (with a repetition rate of $80 \mathrm{MHz}$, a wavelength of $810 \mathrm{~nm}$ and a pulse energy of $0.5 \mathrm{~nJ}$ ). The optical pump reflectance at this interface is 0.5 . The pump light is chopped at a frequency of $1.1 \mathrm{MHz}$ for synchronous lock-in detection. The in-phase transient optical reflectance change at the $\mathrm{Al}_{2} \mathrm{O}_{3}-\mathrm{Cr}$ interface $\delta R(t) / R_{0}$, where $R_{0} \approx 0.6$ is the optical probe reflectance, is measured with frequency-doubled circularly-polarized probe pulses of wavelength $405 \mathrm{~nm}$ and pulse energy $0.06 \mathrm{~nJ}$ as a function of the time delay $t$ between the pump and probe pulses. The pump and probe beams are both focused on the $\mathrm{Al}_{2} \mathrm{O}_{3}-\mathrm{Cr}$ interface with a $50 \times$ microscope objective to provide a lateral resolution of $\sim 1 \mu \mathrm{m}$. The objective is laterally scanned in the plane of the interface to probe different regions of the indented samples.

Fig. 3 shows $\left|\delta R(t) / R_{0}\right|$ (note that $\delta R(t)<0$ ) for the load $F=4.6 \mathrm{~N}$ when probing at the center of the contact (bottom trace) and outside the contact (top trace) for the $215 \mathrm{~nm}$ sample. The $110 \mathrm{~nm}$ sample shows similar features. $\left|\delta R(t) / R_{0}\right|$ takes a lower value for delay times $t<0$ in the case of contact. This is caused by thermallyinduced changes in $\delta R(t) / R_{0}$ at the chopping frequency, discussed in Section 4. At $t=0$ we observe a positive peak due to the excitation and relaxation of hot electrons in the $\mathrm{Cr}$ film. It is followed by a slow decrease owing to heat diffusion. At the maximum delay time we probe, $\tau=150 \mathrm{ps}$, the diffusion length in $\mathrm{Cr}$ is $\mu \sim(2 \kappa \tau / C)^{1 / 2}=$ $94 \mathrm{~nm}$, where $\kappa=94 \mathrm{~W} \mathrm{~m}^{-1} \mathrm{~K}^{-1}$ and $C=3.2 \times 10^{6} \mathrm{~J} \mathrm{~m}^{-3} \mathrm{~K}^{-1}$ are 


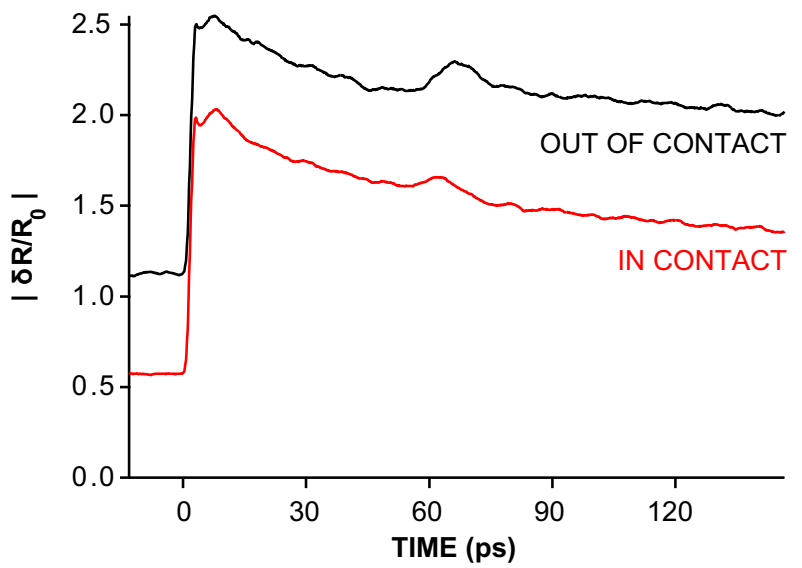

Fig. 3. $\left|\delta R(t) / R_{0}\right|$ for the load $F=4.6 \mathrm{~N}$ when probing at the center of the contact (bottom trace) and outside the contact (top trace) in the $215 \mathrm{~nm}$ sample.

the thermal conductivity and heat capacity of single crystal $\mathrm{Cr}$, respectively [11]. Since $\mu$ is smaller than the film thickness, the picosecond-time-scale temperature variation at the contacting interface is not significant. Indeed in experiment, the background thermal decay does not depend on the contact (see Fig. 3). In what follows, we therefore do not consider picosecond-time-scale heat flow in the analysis of the mechanical contact.

Initially a hydrostatic longitudinal stress field is generated in the $\mathrm{Cr}$ over a depth $\sim 20 \mathrm{~nm}$ determined by the $\sim 15 \mathrm{~nm}$ optical absorption depth [20] at the pump wavelength and by the $\sim 15 \mathrm{~nm}$ electron diffusion depth [11]. The acoustic impedances of $\mathrm{Cr}$ and $\mathrm{Al}_{2} \mathrm{O}_{3}$ are such that the acoustic strain reflection coefficient at the film-substrate interface is almost zero. This results in two unipolar compressive longitudinal acoustic pulses of typical frequency $40 \mathrm{GHz}$ and wavelength $170 \mathrm{~nm}$ being launched perpendicular to the film-substrate interface in opposite directions [21]. The acoustic strain pulse in the $\mathrm{Cr}$ film is reflected from the $\mathrm{Cr}$ film surface (or film-indenter interface), and is detected at the filmsubstrate interface, giving rise to the echoes at $t=\tau \sim 65 \mathrm{ps}$ in Fig. 3 (and at $t=\tau \sim 30 \mathrm{ps}$ in the $110 \mathrm{~nm}$ sample). Only one echo is detected because of the acoustic impedance matching at the $\mathrm{Al}_{2} \mathrm{O}_{3}-\mathrm{Cr}$ interface. The second acoustic pulse is transmitted into the substrate, and produces oscillations in $\delta R(t) / R_{0}$ at the Brillouin frequency $\sim 100 \mathrm{GHz}$ [21]. $\mathrm{Al}_{2} \mathrm{O}_{3}$ has been chosen for its relatively high sound velocity $(11.1 \mathrm{~km} / \mathrm{s})$ and low photoelastic constant $p_{12}(-0.03$ at the probe wavelength) in order to minimize these oscillations. Due to transmission through the film-indenter interface and scattering on the asperities, the height of the acoustic echo is reduced in contact. This phenomenon, analyzed with a proper calibration, allows the determination of the contact pressure $[4,7,8]$.

A distinctive characteristic of picosecond ultrasonics is the ability to extract the nanoscale film indentation $\Delta h=h_{0}-h=$ $h_{0}-V_{f} \tau / 2$ from the echo arrival time $t=\tau$ and the film thickness $h_{0}$ out of contact. Thereby the deformation of the film is monitored during the indentation process. The indentations $\Delta h$ obtained in the $110 \mathrm{~nm}$ and $215 \mathrm{~nm}$ samples are plotted as a function of the radial coordinate $r$ in Fig. 4a and b, respectively. The radius of the indented region increases with load, as described by Eq. (4). The observed spikes show correlation at the different applied loads, which points to the influence of the surface roughness. The indentation profiles in the thicker $\mathrm{Cr}$ film appear to be deeper and more sensitive to the indenter roughness. In order to confirm this observation, further measurements were performed.

The load $F$ is plotted against maximum indentation (at $r=0$ ) in Fig. 5a for a first load-unload cycle (squares and triangles, respec-
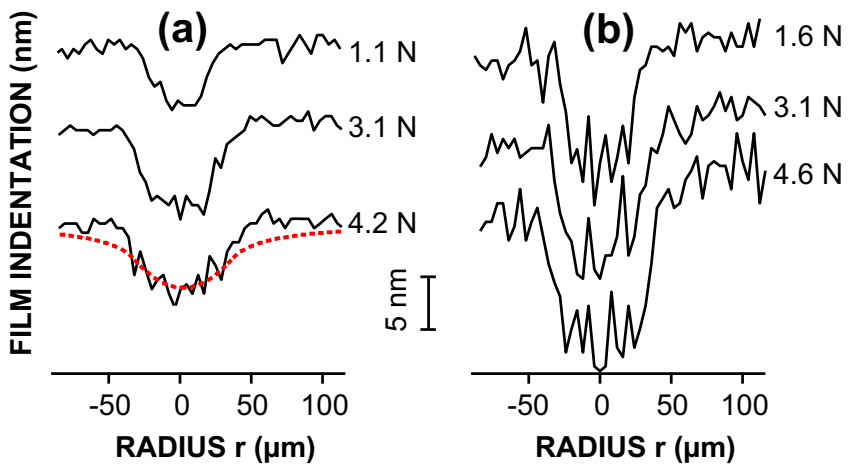

Fig. 4. (a) Film indentation vs. the radial coordinate $r$ at different loads in the $110 \mathrm{~nm}$ sample. Comparison between experiment (solid lines) and theory (dashed line) for $F=4.2 \mathrm{~N}$. (b) Film indentation vs. $r$ in the $215 \mathrm{~nm}$ sample.

tively) in steps lasting $10 \mathrm{~min}$ in the $110 \mathrm{~nm}$ sample. A second load-unload cycle (circles and diamonds, respectively) performed at another location (not previously indented) shows the same trend, demonstrating the reproducibility of the experiment. The micro-hardness $H=19 \mathrm{GPa}$ of RF sputtered $\mathrm{Cr}$ films of thickness $\sim 100 \mathrm{~nm}$ has been determined by Vickers indentation in Ref. [22]. For soft metals, this value corresponds to a yield stress in compression of $\approx 0.36 \mathrm{H}=7 \mathrm{GPa}$ [14]. We estimate the maximum pressure $p_{0}=1.6 \mathrm{GPa}$ at the highest load $F=4.6 \mathrm{~N}$ from Eq. (2). Thus the loading of the film is not sufficient for the bulk of the $\mathrm{Cr}$ to yield. The loading and unloading curves are nearly identical, as expected in the elastic regime.

We now compare the measured indentation $\Delta h$ at $r=0$ with the theory of indentation of a thin film on a rigid substrate by a rigid sphere [23]:

$F=\frac{\pi\left(1-v_{f}\right) R E_{f} \Delta h^{2}}{\left(1+v_{f}\right)\left(1-2 v_{f}\right) h_{0}}$.

As seen in Fig. 5a for the $110 \mathrm{~nm}$ film, this model (dashed line) provides a reasonable estimate of the indentation at the lowest loads $(F<0.5 \mathrm{~N})$. However, the measured $\Delta h$ is smaller than that predicted at the highest loads, owing to the deformation of both the indenter and the substrate. The indentation at $r=0$ in the $215 \mathrm{~nm}$ film corresponding to Fig. $4 \mathrm{~b}$ (averaged over three points in the center of the contact) shows very good agreement with this theory, as depicted in Fig. 5b. This is presumably due to the lesser role played by the indenter and substrate elasticities for the thicker film [24].

To account for the elasticity of the substrate, one must determine the stresses resulting from an axisymmetric static load distribution on a finite region of the free surface, namely, the Boussinesq
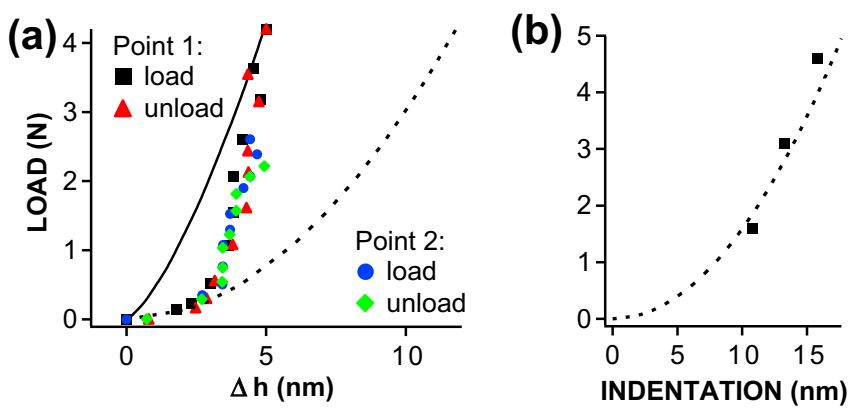

Fig. 5. (a) Maximum indentation measured for load-unload cycles in the $110 \mathrm{~nm}$ sample (solid symbols) compared with theory for a rigid (dashed line) and an elastic (solid line) substrate. (b) Maximum indentation measured in the $215 \mathrm{~nm}$ sample (squares) compared with theory for a rigid substrate (dashed line). 
problem [24,25]. The use of Hankel transforms reduces the formulation of this problem to a system of ordinary differential equations. The detailed solution for an arbitrary pressure distribution is given in Ref. [26]. Here we assume that the pressure distribution $p(r)$ at the contacting interface is not perturbed by the $\mathrm{Cr}$ film and remains Hertzian [27], as described in Eq. (1). The Hankel transform of order zero for $p(r)$ is:

$\tilde{p}(\xi)=\int_{0}^{+\infty} p(r) r J_{0}(\xi r) d r=p_{0}\left[-\frac{\cos (\xi a)}{\xi^{2}}+\frac{\sin (\xi a)}{a \xi^{3}}\right]$

where $J_{0}$ is the zeroth order Bessel function of the first kind. Introducing Eq. (8) into the explicit expressions given in Ref. [26] allows us to calculate the $\mathrm{Cr}$ film indentation $\Delta h$. We compare this result (solid line) with the measured $\Delta h$ in Fig. 5a. This second approach provides an asymptotic description of the indentation at high loads, when the influence of the substrate's elasticity is greatest. For this reason, it cannot be applied to describe the indentation of the $200 \mathrm{~nm}$ film since the high load limit is not reached for the range of loads we used for this sample. To represent $\Delta h$ over the whole range of loads applied, the increasing participation of the substrate with increasing load should be introduced.

The second model for the indentation of an elastic layer resting on an elastic substrate also describes the radial deformation of the film. Since the predicted indentation matches the data well for the $110 \mathrm{~nm}$ film at high loads, we compare the indented profiles for the load $F=4.2 \mathrm{~N}$ in Fig. 4a. Good agreement is observed. When comparing with theory, the measured profile seems to bulge slightly at the edges of the contact area. This suggests the pressure distribution at the contacting interface is modified by the presence of the film.

\section{MHz thermoreflectance}

We also image the contact area in two spatial dimensions over a $200 \times 200 \mu \mathrm{m}^{2}$ region with step $1 \mu \mathrm{m}$, as shown for the example of $t=40 \mathrm{ps}$ for the $110 \mathrm{~nm}$ sample in Fig. 6. Newton's rings [28] are visible owing to the modulated optical absorption at the pump wavelength $\lambda_{p p}=810 \mathrm{~nm}$. They appear at a spacing determined by thickness variations $\Delta d$ of the ball-film air gap for which

$\Delta d=\frac{m \lambda_{p p}}{2}$

for the $m$ th ring. Thereby we measure $\Delta d$ outside the contact area.

It is important to check if substrate bending affects these measurements. For a substrate of thickness $h_{s}=0.8 \mathrm{~mm}$ and for a sample holder pierced with a hole of radius $a_{h}=1.8 \mathrm{~mm}$, plate theory can be applied [29]. The flexural rigidity of the sample is given by

$D=\frac{E_{s} h_{s}^{3}}{12\left(1-v_{s}^{2}\right)}$.

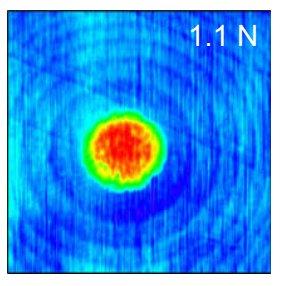

$200 \mu \mathrm{m} \rightarrow$
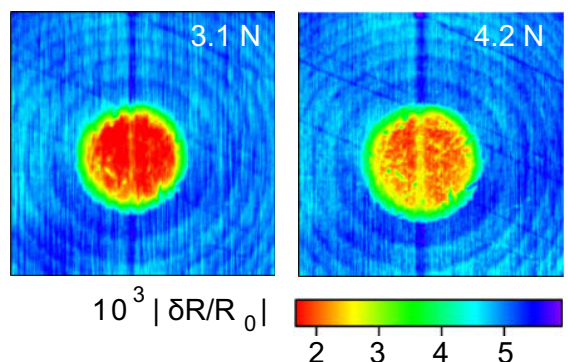

Fig. 6. $\left|\delta R(t) / R_{0}\right|$ images of the contact at $t=40 \mathrm{ps}$ for the $110 \mathrm{~nm}$ sample.
Solving the equations of equilibrium for a simply supported circular geometry under a concentrated force $F$ applied at $r=0$ leads to the following expression for the maximum deflection at the center of the sample:

$h_{d}=\frac{F a_{h}^{2}}{16 \pi D} \frac{3+v_{s}}{1+v_{s}}$.

For the maximum load $F=4.6 \mathrm{~N}, h_{d}=40 \mathrm{~nm}$. Since $h_{d} \ll a_{h}$, the curvature of the sample is negligible.

We therefore calculate the gap predicted by Hertz theory for a $\mathrm{Al}_{2} \mathrm{O}_{3}$ half-space. The $\mathrm{Cr}$ layer is not considered since its deformation, measured at $\sim 5 \mathrm{~nm}$, is negligible compared to that of sapphire: $\delta_{0} \approx 500 \mathrm{~nm}$ at the maximum load $F=4.6 \mathrm{~N}$. When the load $F$ is applied, both the sphere and the sample are deformed. The sum of the deformations of each solid is

$\delta=\frac{\pi p_{0}}{4 E^{*} a}\left(2 a^{2}-r^{2}\right)$

in contact, and

$\delta=\frac{p_{0}}{2 E^{*} a}\left[\left(2 a^{2}-r^{2}\right) \sin ^{-1}\left(\frac{a}{r}\right)+a r \sqrt{1-\frac{a^{2}}{r^{2}}}\right]$

out of contact [14]. The position of the sphere surface during loading is described by the equation of the sphere penetrating into the sample by a depth $\delta_{0}$ :

$d_{0}=R-\sqrt{R^{2}-r^{2}}-\delta_{0}$.

During loading, the gap thickness becomes

$\Delta d=d_{0}-\delta$.

The position of the indenter and the deformed surface of the sample are shown in Fig. 7a. We compare the predicted gap thickness with the measurement from the Newton's rings. The result for $F=3.1 \mathrm{~N}$ is plotted in Fig. 7b. Good agreement is found, confirming that the sample bending does not significantly affect the indentation process for $F<4.6$ N. Fig. 8 shows the thermoreflectance image data for the $215 \mathrm{~nm}$ sample. As predicted for thicker films, no residual pump light penetrates through the $\mathrm{Cr}$ in the air gap, and so no Newton's rings are observed.

An offset is observed in the transients in Fig. 3 due to the modulated temperature change $\delta T$ at the chopping frequency. This thermoreflectance contribution dominates the variation observed in Figs. 6 and 8. The contact size increases with $F$, in accord with Hertz theory [14]. Results for $\left|\delta R(t) / R_{0}\right|$ in both samples are compared in Fig. 9 at $t=-10 \mathrm{ps}$, a time when only the thermal effects contribute. Due to the use of laser pulse trains, thermal harmonics of the laser repetition frequency also have an influence at the chopping frequency [30]. The normalized amplitude $A(r)$ of $\delta T$ is shown. The contrast between in and out of contact is similar for both sam-
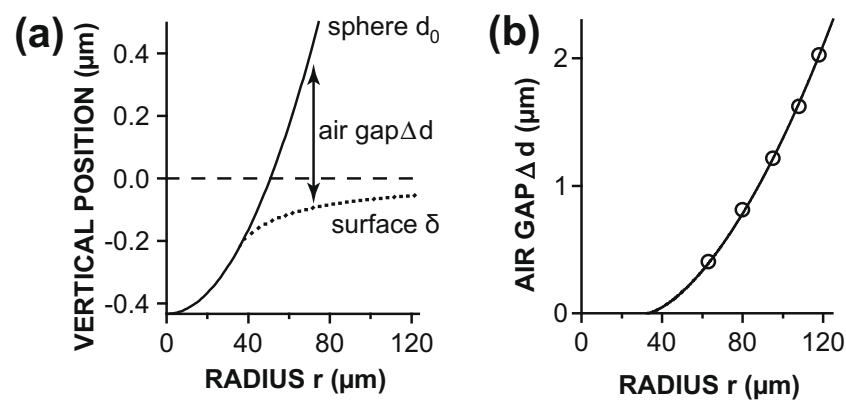

Fig. 7. (a) Position of the $\mathrm{Si}_{3} \mathrm{~N}_{4}$ sphere (solid line) and the deformed sample surface (dotted line). (b) Measured air gap thickness (circles) compared with Hertz theory (line). 

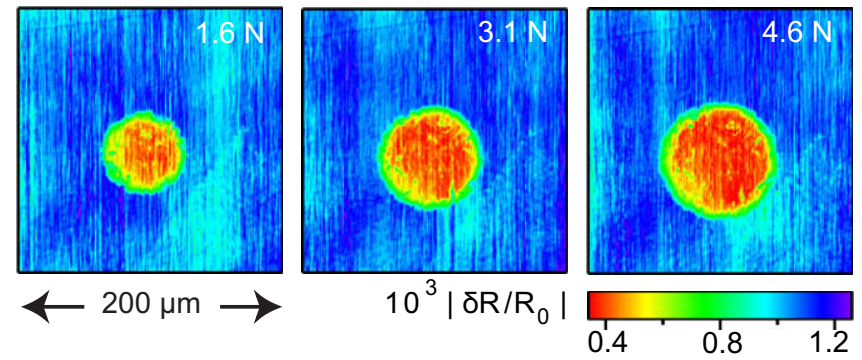

Fig. 8. $\left|\delta R(t) / R_{0}\right|$ images of the contact at $t=55 \mathrm{ps}$ for the $215 \mathrm{~nm}$ sample.
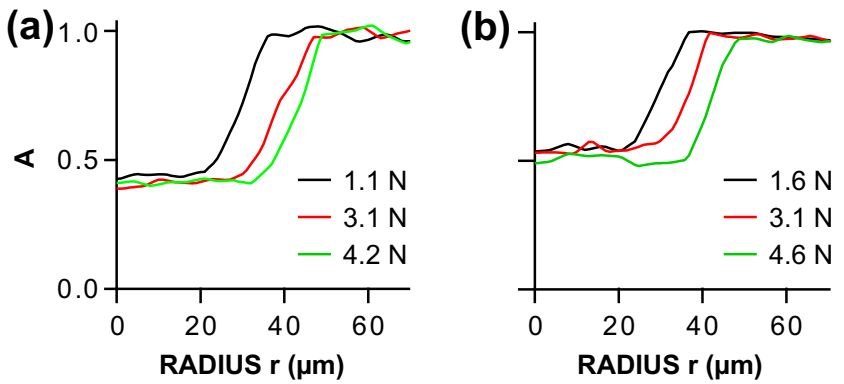

Fig. 9. Normalized amplitude of $\left|\delta R(t) / R_{0}\right|$ for $t<0$ in (a) the $110 \mathrm{~nm}$ sample and (b) the $215 \mathrm{~nm}$ sample.

ple thicknesses. $\delta T$ remains constant in the contact area, and is independent of load $F$. Yet for non-interacting asperities, overall force balance dictates that $S_{r} / S_{0}=p_{0} / H$, where $S_{0}$ is the apparent contact area described by Hertz theory and $S_{r}$ is the real contact area formed by the contacting asperities [31]. As $S_{r}$ increases with increasing $F$, one might expect the contact thermal boundary resistance and $\delta T$ to decrease in contact as $F$ increases [31]. However, the observed $F$-independent thermal contrast suggests that this boundary resistance does not depend significantly on load over the range of loads probed. This anomalous behaviour could be explained by an enhanced heat transport through the interstitial air gaps. It might alternatively arise from the peculiarities of the three-dimensional solution of the heat diffusion equation for a system involving thermal boundary resistances at the film-substrate and film-indenter interfaces. The thermal measurement of the contact should offer a high sensitivity to the presence of the contact at lower $F$, whereas the acoustic measurements are more suitable at higher $F$.

\section{Conclusion}

In conclusion, we have applied picosecond ultrasonics to probe thin film properties during indentation by a sphere. Access to surface deformation during the indentation process provides a useful tool for investigating nanoscale plastic flow in or near the contact area. In addition, $\mathrm{MHz}$ thermoreflectance gives clear images of the thermal contact. In future, it would be interesting to extend the method to the study of films thinner than the picosecond-timescale thermal diffusion length to probe high frequency heat transport through imperfect nanoscale interfaces. The use of an acousti- cally mismatched film and substrate should allow the acoustic reflection from the indenter-film interface to be studied in more detail.

\section{References}

[1] G.M. Pharr, W.C. Oliver, Measurement of thin film mechanical properties using nanoindentation, Mater. Res. Soc. Bull. 17 (1992) 28-33.

[2] N.A. Burnham, R.J. Colton, Measuring the nanomechanical properties and surface forces of materials using an atomic force microscope, J. Vac. Sci. Technol. A 7 (1989) 2906-2913.

[3] M.V. Swain, Mechanical property characterisation of small volumes of brittle materials with spherical tipped indenters, Mater. Sci. Eng. A 253 (1998) 160166.

[4] M. Masuko, I. Yoshimi, Measurement of contact pressures by means of ultrasonic waves, Ann. CIRP 17 (1969) 289-296.

[5] K. Kendall, R.F.S. Tabor, An ultrasonic study of the area of contact between stationary and sliding surfaces, Proc. R. Soc. Lond. A 323 (1971) 321-340.

[6] B.W. Drinkwater, R.S. Dwyer-Joyce, P. Cawley, A study of the interaction between ultrasound and a partially contacting solid-solid interface, Proc. R. Soc. Lond. A 542 (1996) 2613-2628.

[7] A.M. Quinn, B.W. Drinkwater, R.S. Dwyer-Joyce, The measurement of contact pressure in machine elements using ultrasounds, Ultrasonics 39 (2002) 495502.

[8] T. Dehoux, O.B. Wright, R. Li Voti, V.E. Gusev, submitted for publication.

[9] C. Thomsen, H.T. Grahn, H.J. Maris, J. Tauc, Surface generation and detection of phonons by picosecond light pulses, Phys. Rev. B 34 (1986) 4129-4138.

[10] O.B. Wright, K. Kawashima, Coherent phonon detection from ultrafast surface vibrations, Phys. Rev. Lett. 69 (1992) 1668-1671

[11] T. Saito, O. Matsuda, O.B. Wright, Picosecond acoustic phonon pulse generation in nickel and chromium, Phys. Rev. B. 67 (2003) 205421-205427.

[12] From Toshiba Materials Co. datasheet, 2008.

[13] N. Yu, A.A. Polycarpou, Contact of rough surfaces with asymmetric distribution of asperity heights, J. Tribol 124 (2002) 367-376.

[14] K.L. Johnson, Contact Mechanics, Cambridge University Press, 1985.

[15] J.A. Greenwood, J.B.P. Williamson, Contact of nominally flat surfaces, Proc. R. Soc. Lond. A 295 (1966) 300-319.

[16] Y. Kimura, Estimation of the number and the mean area of real contact points on the basis of surface profiles, Wear 15 (1970) 47-55

[17] M.F. Doerner, W.D. Nix, A method for interpreting the data from depth-sensing indentation instruments, J. Mater. Res. 1 (1986) 601-609.

[18] R.B. King, Elastic analysis of some punch problems for a layered medium, Int. J. Solid Struct. 23 (1987) 1657-1664.

[19] H.Y. Yu, S.C. Sanday, B.B. Rath, The effect of substrate of the elastic properties of films determined by the indentation test - axisymmetric Boussinesq problem, J. Mech. Phys. Solid 38 (1990) 745-764.

[20] D. Lide, CRC Handbook of Chemistry and Physics, CRC Press, Boca-Raton, 1999.

[21] O.B. Wright, Laser picosecond acoustics in double-layer transparent films, Opt. Lett 20 (1995) 632-634.

[22] W. Lu, K. Komvopoulos, Nanomechanical and nanotribological properties of carbon, chromium, and titanium carbide ultrathin films, J. Tribol. 123 (2001) 717-724.

[23] M.J. Jaffar, A general solution to the axisymmetric frictional contact problem of a thin bonded elastic layer, Proc. Inst. Mech. Eng. 211 (1997) 549-557.

[24] H.Y. Yu, S.C. Sanday, B.B. Rath, The effect of substrate on the elastic properties of films determined by the indentation test - axisymmetric Boussinesq problem, J. Mech. Phys. Solid 38 (1990) 745-764.

[25] J.W. Harding, I.N. Sneddon, The elastic stresses produced by the indentation of the plane surface of a semi-infinite elastic solid by a rigid punch, Proc. Camb. Phil. Soc. 41 (1945) 16-26.

[26] J. Li, T.-W. Chou, Elastic field of a thin-film/substrate system under an axisymmetric loading, Int. J. Solid Struct. 34 (1997) 4463-4478.

[27] S. Math, V. Jayaram, S.K. Biswas, "Deformation and failure of a film/substrate system subjected to spherical indentation: Part I. Experimental validation of stresses and strains derived using Hankel transform technique in an elastic film/substrate system, J. Mater. Res. 21 (2006) 774-782.

[28] A. Cameron, R. Gohar, Theoretical and experimental studies of the oil film in lubricated point contact, Proc. R. Soc. Lond. A 291 (1966) 520-536.

[29] S. Timoshenko, Theory of Plates and Shells, McGraw-Hill, 1940.

[30] A.J. Schmidt, X. Chen, G. Chen, Pulse accumulation, radial heat conduction, and anisotropic thermal conductivity in pump-probe transient thermoreflectance, Rev. Sci. Instrum. 79 (2008) 114902.

[31] C.V. Madhusudana, Thermal Contact Conductance, Springer, New York, 1996. 